\title{
Development of single frame X-ray framing camera for pulsed plasma experiments
}

\author{
J UPADHYAY*, J A CHAKERA, C P NAVATHE, P A NAIK, \\ A S JOSHI and P D GUPTA \\ Laser Plasma Division, Centre for Advanced Technology, P O CAT, Indore 452013 \\ *e-mail: janky@cat.ernet.in
}

MS received 5 January 2006; revised 27 March 2006

\begin{abstract}
A single-frame X-ray framing camera has been set up for fast imaging of X-ray emissions from pulsed plasma sources. It consists of two parts, viz. an $\mathrm{X}$-ray pin-hole camera using an open-ended microchannel plate (MCP) detector coupled to a CCD camera, and a high voltage short duration gate pulse for the MCP. The camera uses a $10-\mu \mathrm{m}$ pin-hole aperture for imaging on the MCP detector with a magnification of $6 \mathrm{X}$. The high voltage pulser circuit generates a pulse of variable duration from 5 to $30 \mathrm{~ns}$ (at 70\% of peak amplitude) with variable amplitude from $800 \mathrm{~V}$ to $1.25 \mathrm{kV}$, and is triggered through a laser pulse synchronized with the event to be recorded. The performance of the system has been checked by recording $\mathrm{X}$-ray emission from a laser-produced copper plasma. A reduction factor of $\sim 6.5$ is seen in the dark current contribution as the MCP gate pulse is decreased from $250 \mu$ s to $5 \mathrm{~ns}$ duration.
\end{abstract}

Keywords. Microchannel plate; X-ray framing; high voltage pulser.

\section{Introduction}

In view of the poor availability of reflective as well as diffractive X-ray optics, imaging of $\mathrm{X}$-ray emission from pulsed plasma sources is primarily carried out using a pin-hole camera. The advantages of an X-ray pin-hole camera are ease of fabrication, simple operation, and its ability to provide moderately high spatial resolution pictures. Measurements of X-ray emission and mapping of different density and temperature regions in the plasma can be carried out by using different X-ray filters. High-speed gated X-ray imaging is desirable to obtain time-resolved information in a variety of research investigations such as laser plasma interaction, capillary discharge plasma, plasma focus, tokamak devices etc. In principle, this can be accomplished by using an X-ray framing camera with multiframe recording facility. The latter necessitates use of a stripline microchannel plate (MCP) with an array of pinholes (Bradley et al 1992), where a high-voltage gate pulse travels along the stripline, recording each pinhole image sequentially on a phosphor screen. Such stripline MCPs are very expensive and not easily available commercially. As a simpler alternative, we can use a regular MCP with a single pin-hole and activate it with a short-duration gating pulse to record a single 
frame, and obtain X-ray images in consecutive time frames by delaying the gate pulse from the event in multiple plasma shots. Gating of the camera for a few nanoseconds duration has the added advantage that it can improve signal-to-noise ratio significantly. During detection and imaging of the transient events of a few nanoseconds, if there is no gate pulse at the detector, dark current contribution gets integrated over $20 \mathrm{~ms}$ duration of a single frame of the CCD camera, resulting in poor $S / N$ ratio. Since the gate pulse applied to the camera is of much shorter duration, the $S / N$ ratio gets improved. Gating of the MCP is also desirable for longer operational life of the MCP.

In this paper, we describe the development of a single-frame X-ray framing camera using an MCP detector, the design of a short-duration, high-voltage gate pulser circuit, and results of the system performance by imaging X-ray emission from plasma produced by focusing a $30 \mathrm{~J}, 2$ ns (FWHM) Nd:glass laser on a copper target.

\section{System description}

A schematic diagram of the single frame X-ray framing camera using an X-ray pin-hole camera attached to a plasma chamber, along with its on-line detection system is shown in figure 1. The pin-hole is $10 \mu \mathrm{m}$ aperture and is mounted in of cylindrical housing attached to a flange in a direction making an angle of 90 degrees w.r.t. the target normal. An MCP is used as the detector and is mounted on the flange behind the pin-hole camera; the assembly is coupled to one of the ports of the plasma chamber. For imaging the plasma on the MCP detector, the pin-hole is placed at a distance of $75 \mathrm{~mm}$ from the target and $450 \mathrm{~mm}$ from the $\mathrm{MCP}$ detector. This provides image magnification of $6 \mathrm{X}$.

Microchannel plates (MCP) are secondary electron multipliers used for detection and imaging of XUV-soft X-ray radiation, electrons and ions (Morgan 1985). The MCP used in this

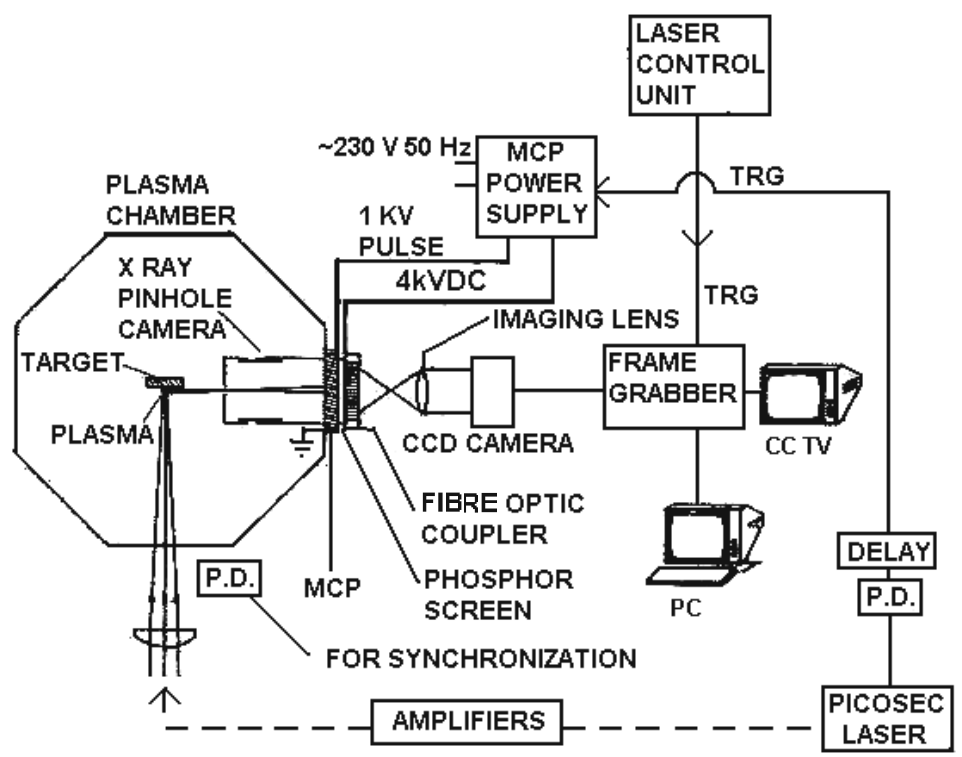

Figure 1. Block diagram of the X-ray framing camera along with experimental setup. 
experiment is a single-stage open-ended detector of $30 \mathrm{~mm}$ diameter. It has a large number of channels, each of $\sim 20 \mu \mathrm{m}$ diameter and $\sim 0.5 \mathrm{~mm}$ length. They are capable of providing high gain $\left(>10^{4}\right)$, 2D-imaging with spatial resolution of $30 \mu \mathrm{m}$, fast time response, and features such as low power consumption, small size, light weight, and immunity to magnetic fields (Hamamatsu Technical Manual 1987). The output impedance of the MCP is $\sim 1000 \mathrm{M} \Omega$. The current gain of the MCP depends upon the applied voltage. A high voltage of $\sim 1 \mathrm{kV}$ is applied across its electrodes, which in turn provides a gain of $\sim 10^{4}$ through secondary electron multiplication. These secondary electrons are then accelerated by applying $4 \mathrm{kV} \mathrm{DC}$ towards the aluminized phosphor screen. The luminous image thus created is then captured by a CCD-frame grabber combination used for on-line analysis. We can shift the dynamic range of the $\mathrm{MCP}$ by changing the amplitude of the gate pulse applied to its electrode as may be required by the peak X-ray intensity of the plasma under detection.

The MCP is activated by a pulser circuit which generates a variable $800 \mathrm{~V}$ to $1.25 \mathrm{kV}$ amplitude pulse with duration variable from 5 to $30 \mathrm{~ns}$ (full width at $3 \mathrm{~dB}$ points) source, triggered by a photodiode-detecting laser oscillator pulse. A high power Nd:glass laser beam (30J, 2ns FWHM) is focused on a planar solid target kept at the centre of a plasma chamber evacuated to $\sim 10^{-6}$ torr. A high density, high temperature plasma is produced having typical dimension of $\sim 100 \mu \mathrm{m}$ diameter. X-rays generated from this plasma are imaged onto the MCP using the X-ray pin-hole camera. A variable delay box (ranging from $0.1 \mathrm{~ns}$ to $70 \mathrm{~ns}$ ) has also been introduced in the triggering path of the high voltage pulser circuit in order to capture X-ray images in consecutive time frames by delaying the gate pulse from the event in multiple laser shots. The MCP output on the phosphor screen is further imaged onto a CCD camera which in turn is controlled by a frame grabber card, which is triggered by the laser control unit, about $400 \mu$ s prior to the laser pulse. A wide variation in amplitude of the high voltage pulser is desirable to analyse the X-ray frames at various MCP gains.

Since the duration of the laser pulse is only $\sim 2 \mathrm{~ns}$, synchronization of this event with the high voltage gate pulse of $5 \mathrm{~ns}$ (full width at $3 \mathrm{~dB}$ points) duration requires temporal adjustment in triggering. This is accomplished by introducing a delay box in the path of the laser pulse from the oscillator triggering the high voltage pulser circuit. By delaying the trigger to the pulser circuit, we can determine the time duration of the X-ray emission. An intensity scan of the recorded image gives the spatial profile of X-ray emission of the source for that time frame.

\subsection{High voltage nanosecond gate pulse generator}

Pulse generators based on transmission lines are widely used in applications where fast and rectangular high voltage pulses are required. There are various configurations such as pulse generators based on reverse-terminated line, Blumlein configuration etc. We have chosen pulse generators based on a self-matched transmission line (Ishii \& Yamada 1985). This circuit is capable of producing a single, high voltage, output pulse with no subsequent reflections, regardless of the value of the load impedance. The amplitude of the pulse can be varied by changing the load impedance and the duration can be varied by changing the length of the line. Figure 2 shows the basic equivalent circuit of the self-matched transmission line. A coaxial transmission line possessing a characteristic impedance of $Z_{0}$ and transit time $\tau$ forms the basic element of this network. The central conductor of the line is connected to the load at one end, whereas the other end is connected to a matching impedance. The connection of the load and the matching resistor at both ends of the transmission line is established by using two fast-synchronized switches. 


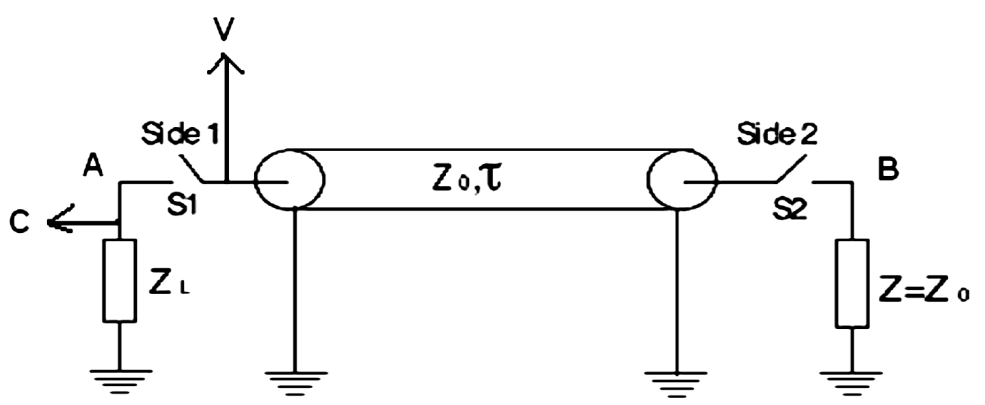

Figure 2. Equivalent circuit of self-matched transmission line pulse generator.

When the transmission line is charged to voltage $V$ and the switches $\mathrm{S}_{1}$ and $\mathrm{S}_{2}$ are closed, the following sequence takes place. As soon as switch $S_{1}$ is closed, the load point (C) attains a potential of $Z_{L} V /\left(Z_{L}+Z_{0}\right)$. As the line is already charged to a voltage $V$, to achieve this potential, a step pulse of $-Z_{0} V /\left(Z_{0}+Z_{L}\right)$ propagates in the line towards $\mathrm{B}$. As the switch $\mathrm{S}_{2}$ is also closed, and the line is terminated at $\mathrm{B}$ by $Z_{0}$, the step pulse gets absorbed at this end with no reflection. At the instant the switch $S_{2}$ is closed, the point $B$ attains a potential of $Z_{0} V /\left(Z_{0}+Z_{0}\right)$ ie $V / 2$. As a result, a step pulse of $-V / 2$ gets launched in the line towards A. This arrives at $\mathrm{A}$ after time $\tau$, and gets reflected at the load. This makes the potential at $\mathrm{C}$ change from $Z_{L} V /\left(Z_{0}+Z_{L}\right)$ to

$$
\left.Z_{L} V /\left(Z_{L}+Z_{0}\right)-V / 2-V / 2 \cdot\left(Z_{L}-Z_{0}\right) / Z_{L}+Z_{0}\right)=0
$$

This means that the point $\mathrm{C}$ is at potential $V Z_{L} /\left(Z_{L}+Z_{0}\right)$ for a time equal to $\left(\tau+\tau_{D}\right)$, where $\tau_{D}$ is the delay between switching on of $S_{2}$ relative to $S_{1}$. Normally both these switches are triggered simultaneously so that $\tau_{D}=0$. The pulse reflected at $\mathrm{C}$ is absorbed at $\mathrm{B}$ as there are no more reflections. Hence the pulse at $\mathrm{C}$ is free from multiple reflections. Thus by using this construction, we can get clean single pulse width upto $\tau$. Pulse amplitude can be changed by changing $Z_{L}\left(V / 2\right.$ for $\left.Z_{L}=Z_{0}\right)$, and pulse width can be changed by changing cable length.

Based on the above configuration, the pulser circuit has been designed as shown in figure 3 . It provides a pulse of variable amplitude and duration in synchronization with the laser pulse. The pulse-forming network consists of a transmission line connected at one end to a load and a matching impedance at the other end through two fast switches. These are made of two avalanche transistor stacks, each of 5 transistors. The transmission line is made of URM-43 co-axial cable having a characteristic impedance of $50 \Omega$. Hence one stack is terminated by a $50 \Omega$, whereas the other stack is terminated by a variable resistor to get a variable output pulse. The avalanche transistors used are 2 N5551, which have nearly similar characteristics, such as breakdown voltage $\sim 320 \mathrm{~V}, h_{\mathrm{fe}} \sim 120$, fall time $\sim 2 \mathrm{~ns}$.

When the line is charged to the voltage of $1.7 \mathrm{kV}$, the lowest transistor of the first and the second stacks are simultaneously triggered through two pulse transformers. Each transformer consists of a toroidal ferrite core and 5 turns of a shielded cable, whose central conductor acts as primary winding, whereas its shield acts as secondary winding. The switching of two stacks in turn launches travelling waves at both ends, propagating through the transmission line. The length of the transmission line is selected through a double pole, 6-way band switch, in order to get a variable pulse width output. A variable load resistor has been used to get variable pulse amplitude. The output pulse can be varied from $800 \mathrm{~V}$ to $1.4 \mathrm{kV}$ pulse. It was 


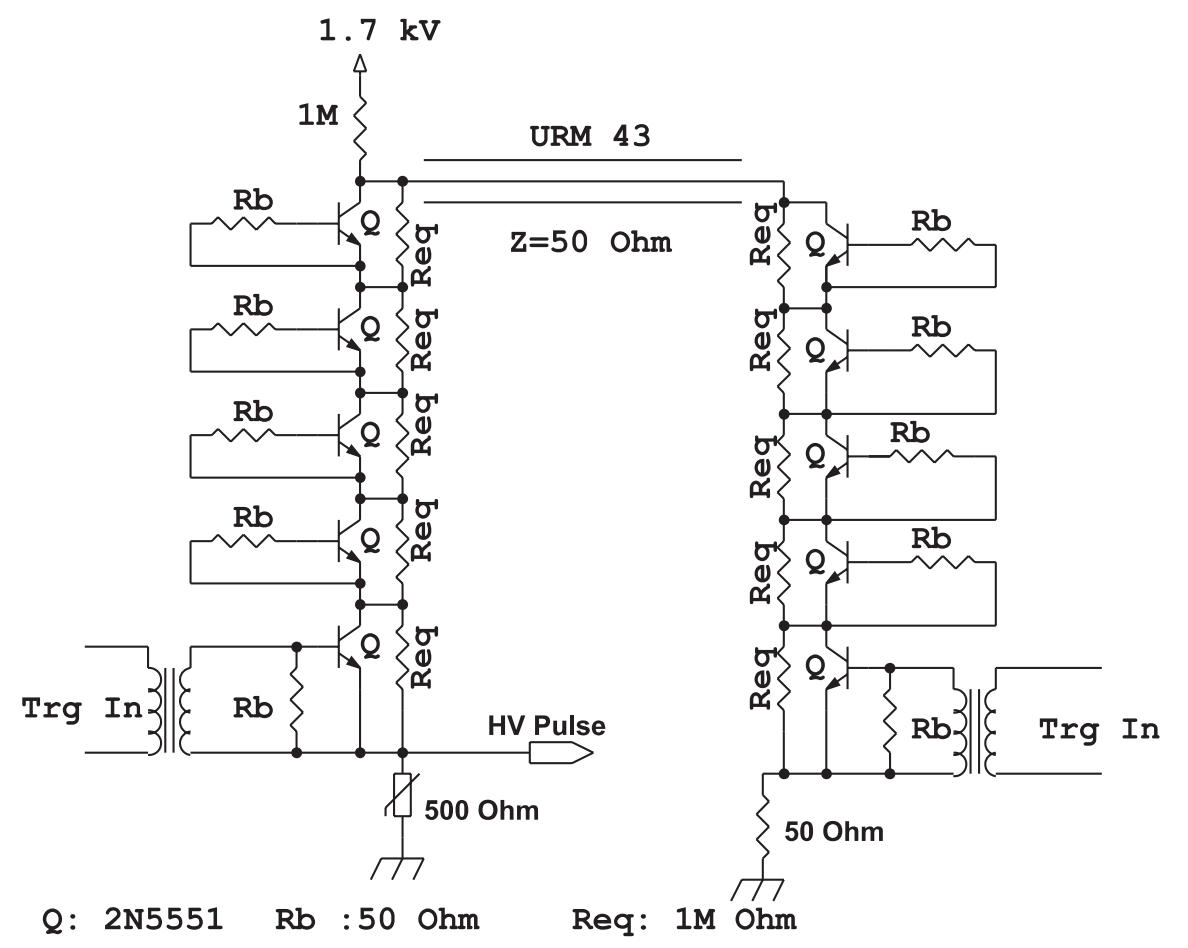

Figure 3. Schematic diagram of the pulser circuit.

observed that both the rise and fall times of the pulse are $\sim 6 \mathrm{~ns}$. The trigger delay was found to be $15 \mathrm{~ns}$ with a jitter of less than $1 \mathrm{~ns}$. Typical output pulses are shown in figure $4 \mathrm{a}, \mathrm{b}$ and $\mathrm{c}$ for $5 \mathrm{~ns}, 7 \mathrm{~ns}$ and $15 \mathrm{~ns}$ duration pulse respectively. It is seen that while an almost flat top pulse is generated in figure $4 \mathrm{~b}$ and $4 \mathrm{c}$ using this circuit, figure $4 \mathrm{a}$ shows a triangular shaped pulse, where flatness is limited by the rise time of the circuit ( $\sim 6 \mathrm{~ns})$. A standard Elnova make $6 \mathrm{kV} / 6 \mathrm{~mA}$ DC supply has been used to bias the phosphor screen to $4 \mathrm{kV} \mathrm{DC}$.

\subsection{Variable delay box}

A variable delay box has been introduced in the path of the trigger input of the high voltage pulser circuit. This delay box provides delay range from 0 to $70 \mathrm{~ns}$, with a resolution of $0.1 \mathrm{~ns}$. It is made using co-axial cable RG-174 having characteristic impedance of $50 \Omega$ and having a propagation delay of $5 \mathrm{~ns} / \mathrm{m}$.

\section{Performance}

The performance of the camera was tested by imaging X-ray emission from plasma produced by focusing a $30 \mathrm{~J}, 2 \mathrm{~ns}$ (FWHM) Nd:glass laser on a copper target. The laser beam from the oscillator section of the laser system after suitable amplification arrives at the plasma chamber. The laser pulse reaching the plasma chamber is detected by a photodiode mounted near the plasma chamber and high voltage pulser, is triggered by the oscillator, and are both synchronized by adjusting the relative delay with the delay box. The laser intensity is about 
(a)

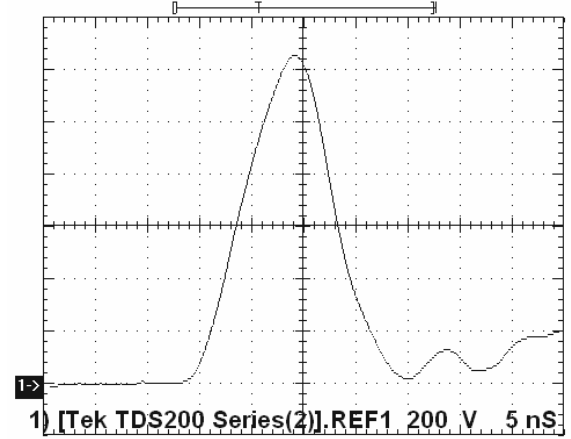

(b)

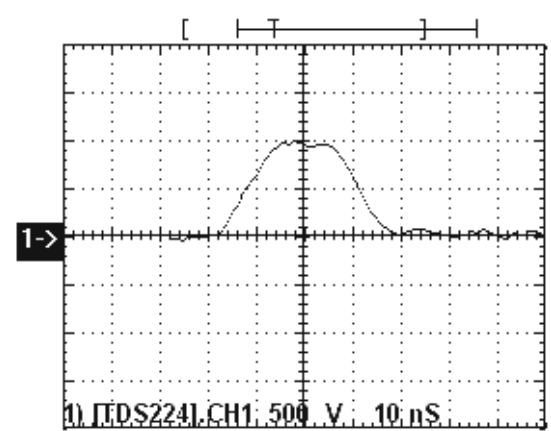

(c)

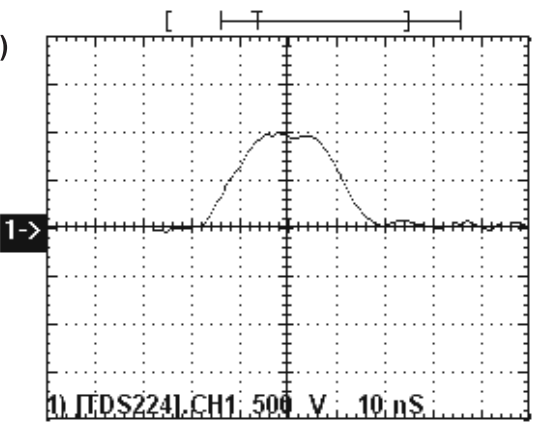

Figure 4. Typical pulses: (a) $1.3 \mathrm{kV} / 5 \mathrm{~ns}$, (b) $1 \mathrm{kV} / 7 \mathrm{~ns}$ pulse and (c) $1 \mathrm{kV} / 15 \mathrm{~ns}$.

$5 \times 10^{13} \mathrm{~W} / \mathrm{cm}^{2}$. An aluminized polycarbonate foil (X-ray cutoff energy $\sim 800 \mathrm{eV}$ ) is placed before the imaging pin-hole. Figures $5 \mathrm{a}, \mathrm{b}$ and $\mathrm{c}$ show the typical X-ray frames captured using this setup. A plasma expansion size of $\sim 100 \mu \mathrm{m}$ (FWHM) is measured, which is in agreement with the focal spot size of the plasma. As the delay of the frame is scanned, it is observed that at a certain delay, the images appear and by increasing it further, the image vanishes. This shows that the $\mathrm{X}$-ray emission lasts for $\sim 5 \mathrm{~ns}$. Figures $5 \mathrm{a}$ and $\mathrm{b}$ show $\mathrm{X}$-ray images of the copper plasma recorded with 5 and $13 \mathrm{~J}$ laser energy respectively, with the same aluminized polycarbonate filter and MCP gain of $1.1 \mathrm{kV} / 7 \mathrm{~ns}$ in both the cases. As we can see, the change in X-ray counts is 48 in the case of the lower laser energy and 182 in the other case. Similarly, figures $5 \mathrm{~b}$ and $\mathrm{c}$ are compared, where the X-ray images are captured with different filters, viz aluminized polycarbonate filter and $7.5 \mu \mathrm{m} \mathrm{Al}$. This analysis shows that X-ray counts reduce from 182 counts to 64 counts. The corresponding intensity versus position profile in the direction of plasma expansion is also shown in the respective figures (figures $5 \mathrm{~b}$ and c). As can be seen from the X-ray frames, X-ray intensity count reduces as we goes from aluminized polycarbonate filter to $7.5 \mu \mathrm{m}$ Al filter for the same laser energy and MCP gain. A significant reduction in the dark current contribution $(S / N$ ratio of 20$)$ is also observed by using $5 \mathrm{~ns}$ gate pulse operation as compared to that recorded using a $250 \mu \mathrm{s}$ gate pulse $(S / N$ ratio of 3$)$ on the MCP detector.

\section{Conclusion}

A single-frame X-ray framing camera has been developed. Using this setup, X-ray images were recorded by gating the MCP with a $1.2 \mathrm{kV} / 5 \mathrm{~ns}$ duration pulse using a pin-hole aperture 
(a)
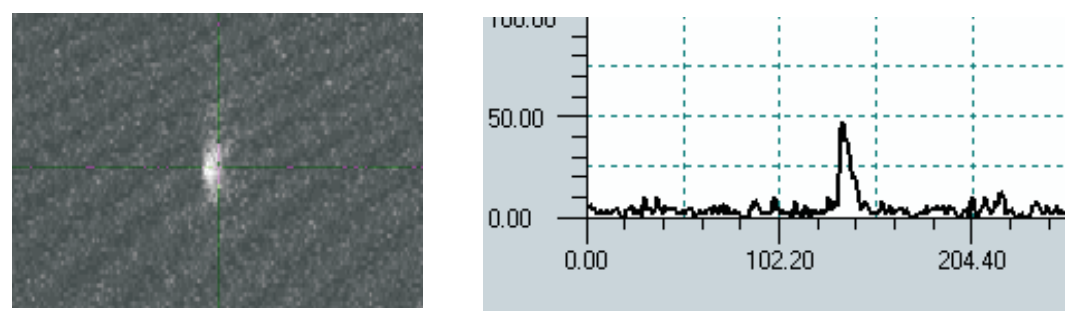

(b)
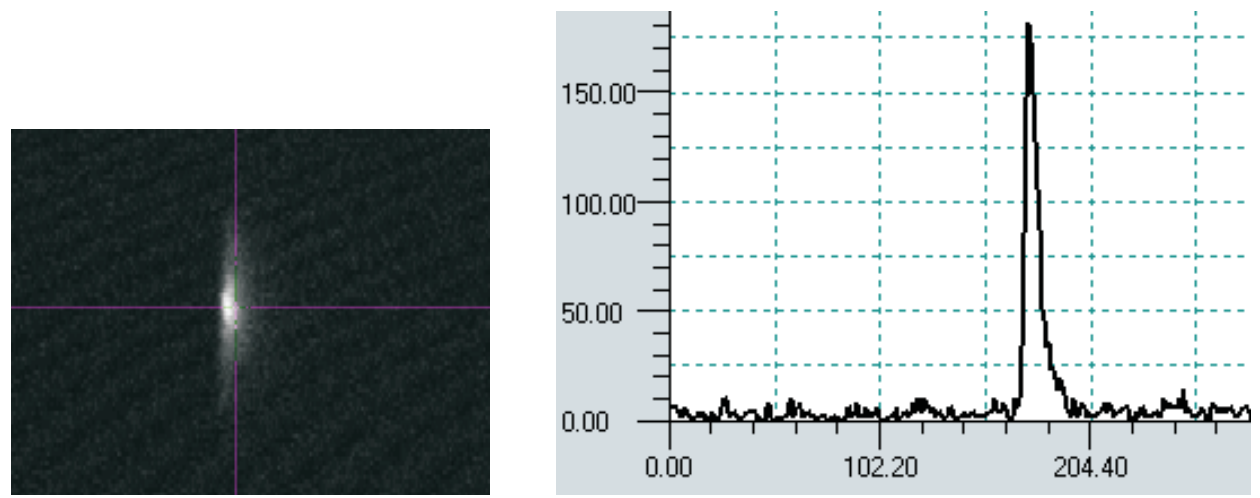

(c)
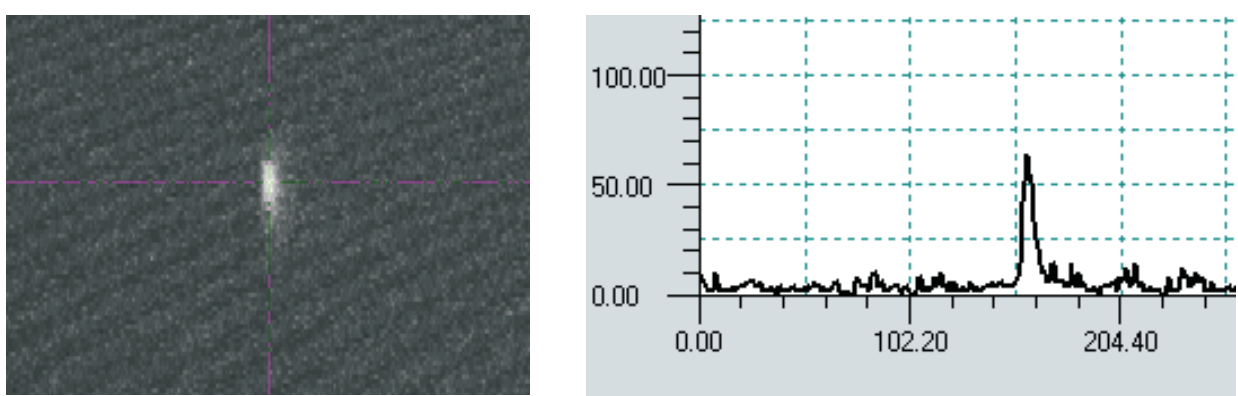

Figure 5. Typical X-ray frames and intensity profiles for (a) $5 \mathrm{~J}$ and (b) $13 \mathrm{~J}$ laser pulse produced $\mathrm{Cu}$-plasma filtered through aluminized polycarbonate filters. (c) A typical X-ray frame and its intensity profile for a 15-J laser pulse produced $\mathrm{Cu}$-plasma filtered through a $7.5 \mu \mathrm{m}$ Al filter.

of size $10 \mu \mathrm{m}$. The X-ray emission region of laser-produced copper plasma filtered through aluminized polycarbonate filter and $7.5 \mu \mathrm{m}$ Al filter have been successfully recorded at different laser intensities. In addition, the high-voltage pulser circuit and its associated units are compact, of low cost, made using indigenously available components, and immune to electromagnetic interference. This system can be used for studying X-ray emission from pulsed plasma devices like capillary discharge and plasma focus, where the event lasts from several tens of nanoseconds to $\sim 100 \mathrm{~ns}$. Framing cameras with frame durations of about $5 \mathrm{~ns}$ can be very useful for analysing such observations. 


\section{References}

Bradley D K, Bell P M, Kilkenny J D, Hanks R, Landen O, Jaanimagi, McKenty P W, Verdon C P 1992 High speed gated X-ray imaging for ICF target experiments. Rev. Sci. Instrum. 63: 4813-4817

Hamamatsu Technical Manual 1987 Characteristics and application of microchannel plates. Res-0795 \& $\mathrm{T}-114$

Ishii M, Yamada H 1985 Self-matched high voltage rectangular wave pulse generator. Rev. Sci. Instrum. 56: $2116-2118$

Morgan B L (ed.) 1985 Photoelectronic images devices (London: Academic Press) 